\title{
Restoration of Maxillary Arch Using Smooth Surface Dental Implants in a Case of Aggressive Periodontitis with Established Crohn's Disease: A Special Case File
}

Abhishek Singh Nayyar*

Department of Oral Medicine and Radiology, Saraswati-Dhanwantari Dental College and Hospital and Post-Graduate Research Institute, India

*Corresponding author: Nayyar AS, Department of Oral Medicine and Radiology, Saraswati-Dhanwantari Dental College and Hospital and Post-Graduate Research Institute, Parbhani, Maharashtra, India, Tel: +91-98509 04067; E-mail: singhabhishekndls@gmail.com

Received: September 11, 2019; Accepted: October 16, 2019; Published: October 25, 2019

\begin{abstract}
An association between periodontitis and various other systemic inflammatory conditions is well-established. Such systemic conditions might, also, influence the clinical outcome in case of implant therapy in periodontitis patients as they might affect the soft tissues, the general trabecular bone pattern as well as affect the successful outcomes of osseo-integration. Crohn's disease is a chronic granulomatous disease process characterized by persistent intestinal inflammation, ulceration, formation of granulomatous lesions and a generalized trans-mural inflammation throughout the intestines. Periodontitis is one of the common oral manifestations of Crohn's disease which was first described in 1969. The present case report describes a case of aggressive periodontitis in a patient with established Crohn's disease successfully managed with implant therapy.
\end{abstract}

Keywords: Periodontitis; Systemic inflammatory condition; Implant therapy; Trabecular bone pattern; Osseo-integration; Inflammatory bowel disease; Crohn's disease; Chronic granulomatous disease process; Intestinal inflammation; Trans-mural inflammation

\section{Introduction}

Periodontal health and disease are dependent on a complex interplay of biofilm-host tissue and immuno-regulation [1]. An association between periodontitis and various other systemic inflammatory conditions is well-established [2]. Such systemic conditions might, also, influence the clinical outcome in case of implant therapy in periodontitis patients as they might affect the soft tissues, the general trabecular bone pattern as well as affect the successful outcomes of osseo-integration [3,4].

Citation: Nayyar AS. Restoration of Maxillary Arch Using Smooth Surface Dental Implants in a Case of Aggressive Periodontitis with Established Crohn's Disease: A Special Case File. Clin Case Rep Open Access. 2019;2(4):137.

(C)2019 Yumed Text. 
www.yumedtext.com | October-2019

Inflammatory bowel disease (IBD) has two components, namely, Crohn's disease and Ulcerative colitis. Crohn's disease is a chronic granulomatous disease process characterized by persistent intestinal inflammation, ulceration, formation of granulomatous lesions and a generalized trans-mural inflammation throughout the intestines [5]. The common oral manifestations of Crohn's disease were first described in 1969 which included gingival hyperplasia, fascicular eruptions, aggressive periodontitis and ulcerations [6]. Flemmig TF, et al. [7] concluded from the findings of their study that patients with inflammatory bowel disease (IBD) had an $11 \%$ higher prevalence rate of periodontitis. Vavricka SR, et al. [8] concluded from their study that markers specific to periodontitis were found in higher percentage in patients affected with chronic inflammatory bowel disease (IBD).

The success of implant therapy depends largely on the integrity of the soft and hard tissues around the dental implants. Nutritional and immune defects along with the soft and hard tissue changes may put Crohn's disease patients at higher risk of implant failure [9]. Alsaadi G, et al. [10] have shown a significant association of Crohn's disease with early implant failure concluding Crohn's disease as one of the most prominent risk factors in such cases. A textbook on dental implants written by Javed F and Romanos GE [11] on dental implants in patients with Crohn's disease in evidence-based implant dentistry and systemic conditions keeps a special emphasis on the risks associated with implant therapy in inflammatory bowel disease cases.

The decision of implant therapy in patients with Crohn's disease, thus, should be taken on a sound clinical evaluation and a thorough analysis of the soft and hard tissues. Failure of dental implants after an initial, short-term success is mainly influenced by the soft tissue behaviour, systemic condition as well as the occlusal forces directed towards the implants. Factors such as occlusal load can be controlled by the operator, so, the main factor influencing implant failure in most of the situations is, largely, governed by the soft tissue behaviour. In patients with inflammatory gingival conditions and a generalized friable gingiva, exposure of the implant surface may be the primary cause of implant failure [12]. The present case report describes such a case of aggressive periodontitis in a patient with established Crohn's disease successfully managed with implant treatment.

\section{Case Report}

The present case report put forths a case of aggressive periodontitis (FIG. 1) in a 36-year old female patient with established Crohn's disease diagnosed since 8 years who reported with pain and mobility in maxillary molar region wherein multiple surgical attempts had been made in order to control the gingival inflammation and who was, later, successfully managed with implant therapy. The patient was on medications including steroid therapy for few years which were gradually tapered with the resolution of her gastro-intestinal symptoms around 3 years back. The patient was, from then, on diet modifications advised to the patient with frequent follow-ups for routine check-ups though without any known complications. She gave a history of bleeding gums with mobility of teeth and recurrent ulcerations since last 5 years. On clinical examination, there was bleeding from the gingiva in the affected region with deep pockets and marked enlargement and superadded inflammatory changes in the said area as well as other areas of the oral cavity. Furthermore, there was generalized mobility in all the teeth except in the mandibular premolar and molar region in third quadrant. There was a history of some kind of dental treatment received in the past for similar reasons though the problem did not subside and the patient continued to have similar problems of generalized bleeding, mobility of the teeth and frequent ulcerations throughout the oral cavity. 
Orthopantomograph (OPG) of the patient revealed a severe generalized bone loss indicative of an aggressive periodontitis with few missing teeth in both the arches. Extraction of all the poor prognosis teeth with grade III mobility followed by implant placement was the line of treatment decided. The probability of peri-implant disease and its evidence was discussed with the patient.

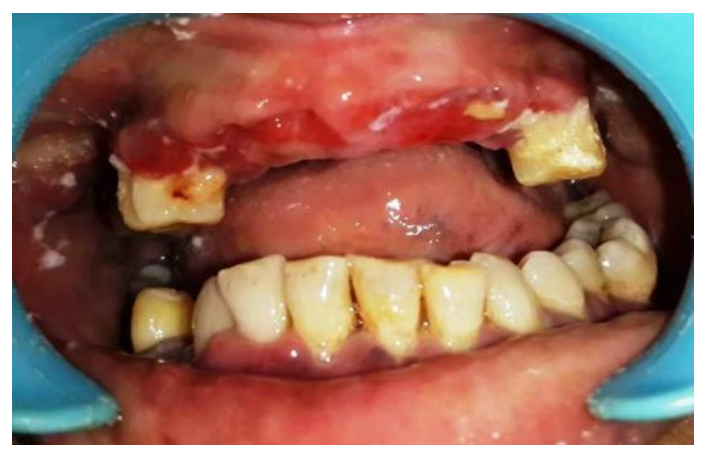

FIG. 1. Clinical picture of the patient with aggressive periodontitis with established Crohn's disease.

The possibility of success with smooth-surfaced implants in the said cases was, also, explained to the patient. After taking patient's consent, it was decided to restore the missing teeth with the help of smooth-surfaced implants with cortical engagement. In order to prevent the complications of uncontrolled tissue shrinkage in the present case, extraction of the anterior teeth was followed by a temporary bridge with the premolars as abutments. It was, also, planned to prevent the placement of implants in maxillary incisor region to provide more prosthetic freedom during the final phase. After a gap of two weeks, surgery was planned. In order to provide an equal occlusal table bilaterally, a cantilever bridge in the lower was planned. The disadvantages of a cantilever bridge were, also, explained to the patient [20]. Due to certain constraints, the patient chose to delay the treatment in the lower arch. On the day of the surgery, the remaining teeth in the maxillary arch were extracted under local anesthesia while the placement of 10 smooth-surfaced implants (Strategic Implant ${ }^{\circledR}$, Simpladent GmbH, Implant type BECES) (FIG. 2a, b, 3) was done based on the principle of cortical engagement. Pterygoid plates, floor of the maxillary sinus and nasal floor were the cortical bones engaged. The placement in the anterior region was oblique (FIG. 4) in order to achieve a better emergence profile and greater prosthetic freedom.

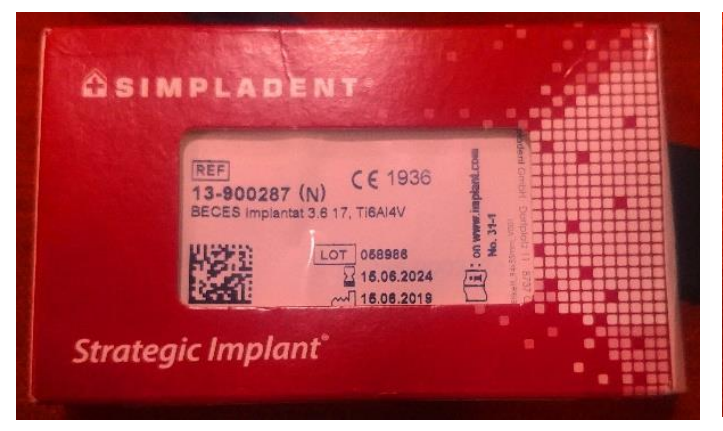

Fig. 2a

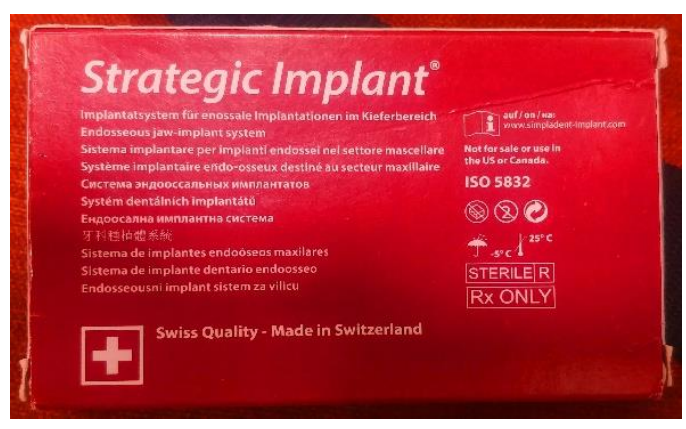

Fig. 2b

FIG. 2a, b. Strategic Implant ${ }^{\circledR}$, Simpladent GmbH, Implant type BECES. 


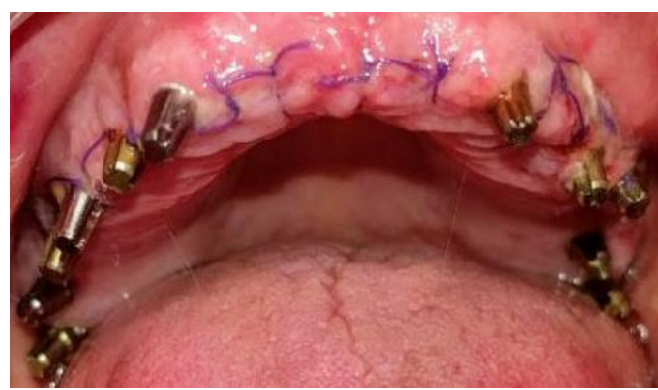

Fig. 3

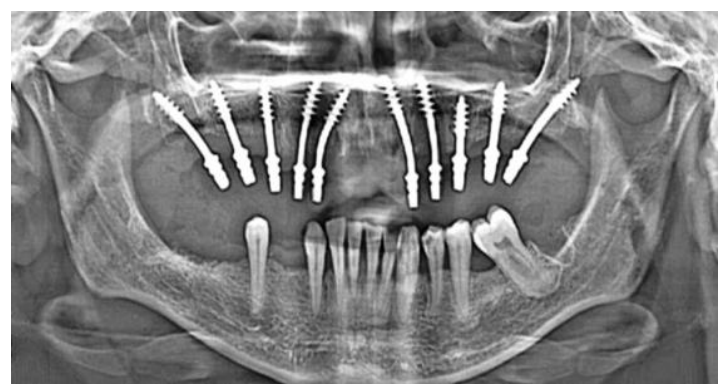

Fig. 4

FIG. 3. Ten smooth-surfaced implants placed in the maxillary arch post-extractions under local anesthesia, FIG. 4.

Orthopantomograph (OPG) of the patient revealing placement of 10 smooth-surfaced implants with oblique placement in anterior region based on the principle of cortical engagement.

Impressions were made according to the manufacturer's instructions using impressions caps and addition silicone material. Laboratory analogs were placed in these impression caps to create a laboratory model. Parallelism of the implant was achieved by bending of the implants during surgery and use of the guiding jig provided by the laboratory technician. A jaw relation was taken in a conventional method using aluminium reinforced bite registration wax. Trial of the metal framework (FIG. 5) was done on the second day. Zig placement guided the operator to eliminate interferences by selective grinding of the implants observed by the technician on the model to facilitate proper placement of the metal framework. After confirming proper placement of the metal framework, registration of jaw relation was done using aluminium reinforced bite registration wax to confirm and improve on to the previously registered jaw relation. A cantilever bridge was designed in the mandible to have equal length of the occlusal table. Avoidance of unilateral chewing which is an important aspect in the philosophy of cortical implantology was taken care of [21]. The final porcelain-fused-to-metal (PFM) bridge (FIG. 6a, b) was cemented on the third day. The occlusal scheme was given according the one described by Ihde S and Ihde AA [21]. The patient was instructed about the importance of bilateral mastication and periodic follow-ups. The follow-up of the case showed stable gingival condition and marked improvement in the consistency and function of gingival tissues (FIG. 7a, b) which showed a noticeable healing. Some amount of expected gingival recession was seen in the posterior regions where implants were placed in the extraction sites. One-year follow-up of the patient showed a stable implant engagement with no cortical radiolucency around the threads of the implants (FIG. 8).

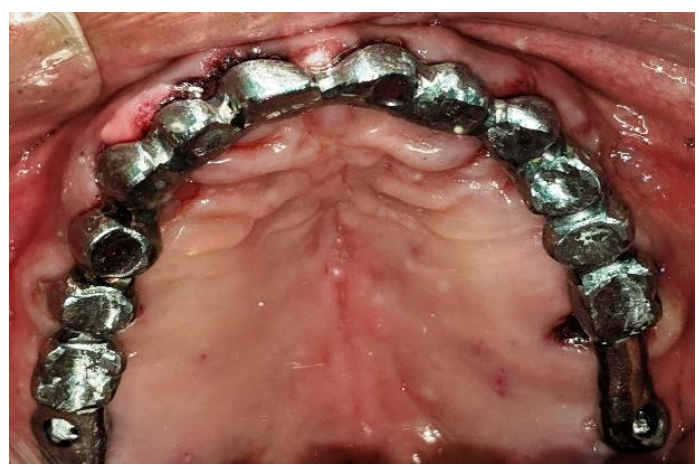

FIG. 5. Trial of the metal framework. 


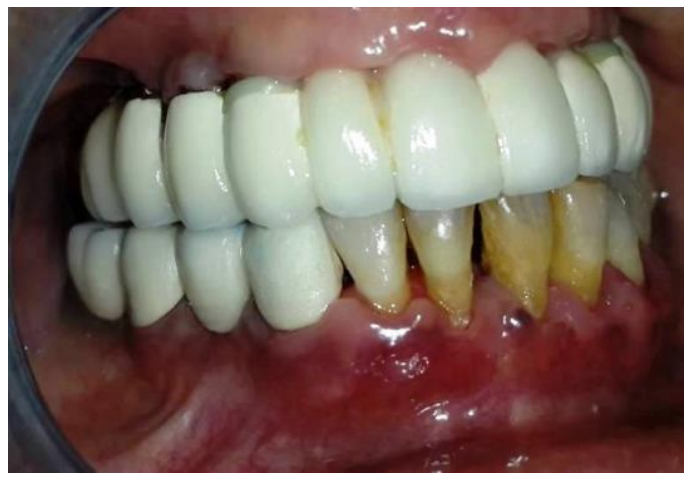

Fig. 6a

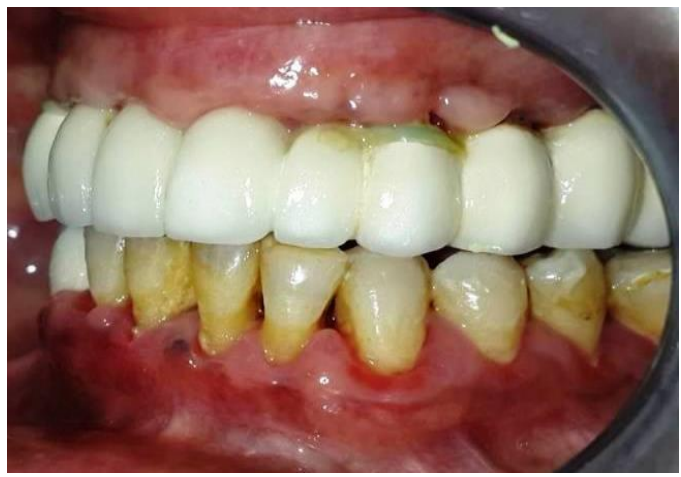

Fig. $6 b$

FIG. 6a, b. Final porcelain-fused-to-metal (PFM) bridge cemented on the third day.

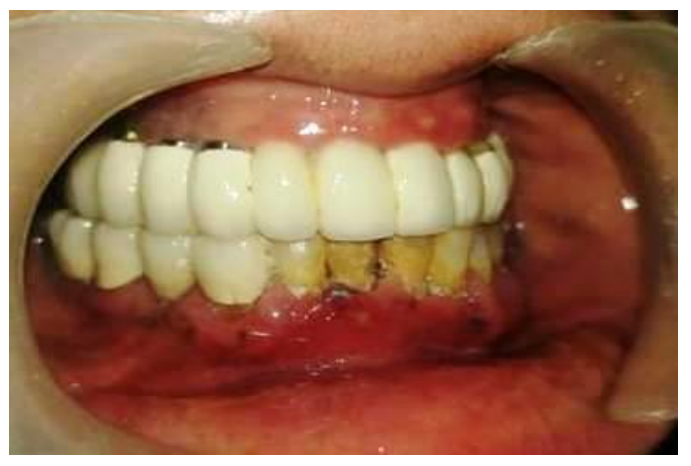

Fig. 7a

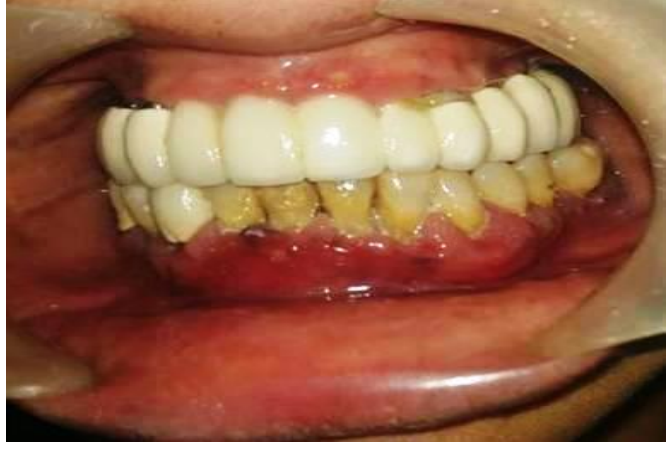

Fig. $7 \mathrm{~b}$

FIG. 7a, b. Stable gingival condition and marked improvement in the consistency and function of gingival tissues showing a noticeable healing on 1-year follow-up of the patient.

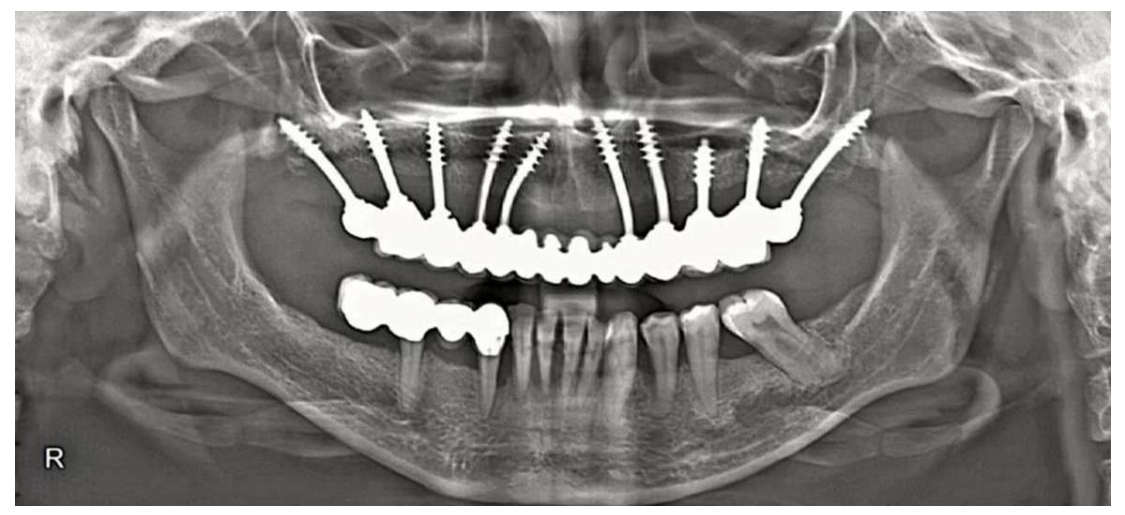

FIG. 8. Orthopantomograph (OPG) of the patient revealing a stable implant engagement with no cortical radiolucency around the threads of the implants on 1-year follow-up of the patient. 
www.yumedtext.com | October-2019

\section{Discussion}

Historically, the implant fixtures used by Branemark and co-workers were machined with relatively smooth-surfaced implants with a two-staged approach [15]. In order to have predictability and an accelerated osseo-integration, surface modifications were adapted on dental implants [16,17]. Unfortunately, year by year, the prevalence of peri-implantitis cases reported in the literature increased alarmingly and such prevalence was found to be between $11.3 \%$ and $47.1 \%$ [18,19]. A meta-analysis published on the association of periodontitis and peri-implantitis cases concluded that subjects with periodontitis are at 2.15 times higher risk for peri-implantitis compared to the healthy subjects [20]. In a survey conducted on the prevalence of peri-implant mucositis in peri-implantitis cases, a prevalence of up to $25 \%$ of peri-implant mucositis in cases with peri-implantitis was recorded while it was found that up to $10 \%$ of the implants were removed due to periimplantitis in such cases [21].

Furthermore, Baron M, et al. [22] concluded from their study that ligature-induced peri-implant disease demonstrated greater bone loss in implants with rough surface compared with the machine-surfaced implants. Also, the latter exhibited better resolution of the disease after the peri-implant ligature was removed. These findings, further, augment the belief that smoothsurfaced implants are more resistant to the peri-implant disease processes while the said study, also, concluded that surface roughness plays no role in the initiation of peri-implant disease, although, it might affect its progression and treatment outcome once the disease is established. Once established, peri-implantitis has a progressive nature with an almost uncertain prognosis.

Esposito M, et al. [23], also, conducted a meta-analysis to evaluate the frequency of peri-implantitis in machine-surfaced implants compared to those with roughened surfaces with a 3-year follow-up wherein three randomized controlled trials were included and concluded that peri-implantitis was significantly less in smooth-surfaced implants compared to implants with roughened surfaces.

Though placement of implants in patients with history of periodontitis is a viable option for oral rehabilitation, the failure rate is significantly higher in such cases when compared to the healthy individuals [24]. In the present case, too, the possibility of peri-implant disease process was discussed with the patient and only after taking consent of the patient, the use of smoothsurfaced implants with cortical engagement for anchorage was decided.

The selected implant design allowed the operator to perform a flapless procedure which has been documented to have significantly left less post-operative discomfort to the patient as well as minimal soft tissue manipulation [25]. Rigid splinting with the help of prosthesis along with cortical engagement, also, permitted the operator to immediately place and load these implants, a technique which, too, has been well-documented in the literature [26,27].

Thus, looking at the literature evidence and the probability of implant supporting structure loss with surface-treated implants, the concept of re-introducing machined, smooth-surfaced implants in the dental practice shows some promise. As these implants engage the resorption-free cortical bone for anchorage, the necessity of bone augmentation procedures can, also, be obviated [26]. 
www.yumedtext.com | October-2019

\section{Conclusion}

Systemic conditions pose a major threat in the success of implant therapy. Also, exposure of the treated implant surfaces provides a suitable bed for the pathogens to proliferate leading to a higher chance of peri-implantitis and implant failure. Many attempts have been made in this direction but the prevalence of peri-implant disease is well-documented. Thus, looking at the literature evidence and the probability of implant supporting structure loss with surface-treated implants, the concept of re-introducing machined, smooth-surfaced implants, especially, in systemically compromised patients, in the dental practice, shows promise. Untreated, smooth-surfaced implants anchored with the help of stable cortical engagement may provide a stable, long-term solution in such cases.

\section{REFERENCES}

1. Chickanna R, Prabhuji M, Nagarjuna M. Host-bacterial interplay in periodontal disease. J Int Clin Dent Res Organ. 2015;7(1):44-50.

2. El-Shinnawi U, Soory M. Associations between periodontitis and systemic inflammatory diseases: Response to treatment. Recent Pat Endocr Metab Immune Drug Discov. 2013;7(3):169-88.

3. Alsaadi G, Quirynen M, Michiles K, et al. Impact of local and systemic factors on the incidence of failures up to abutment connection with modified surface oral implants. J Clin Periodontol. 2008;35(1):51-7.

4. Peron C, Javed F, Romanos GE. Trabecular Metal Implants: A report of two clinical cases and literature review. J Osseointegr. 2015;7:1-5.

5. Zhang YZ, Li YY. Inflammatory bowel disease: Pathogenesis. World J Gastroenterol. 2014;20(1):91-9.

6. Lankarani KB, Sivandzadeh GR, Hassanpour S. Oral manifestation in inflammatory bowel disease: A review. World J Gastroenterol. 2013;19(46):8571-9.

7. Flemmig TF, Shanahan F, Miyasaki KT. Prevalence and severity of periodontal disease in patients with inflammatory bowel disease. J Clin Periodontol. 1991;18(9):690-7.

8. Vavricka SR, Manser CN, Hediger S, et al. Periodontitis and gingivitis in inflammatory bowel disease: A casecontrol study. Inflamm Bowel Dis. 2013;19(13):2768-77.

9. Pranskunas M, Poskevicius L, Juodzbalys G, et al. Influence of Peri-Implant Soft Tissue Condition and Plaque Accumulation on Peri-Implantitis: A Systematic Review. J Oral Maxillofac Res. 2016;7(3):e2.

10. Alsaadi G, Quirynen M, Komárek A, et al. Impact of local and systemic factors on the incidence of oral implant failures up to abutment connection. J Clin Periodontol. 2007;34(7):610-7.

11. Javed F, Romanos GE. Dental Implants in Patients with Crohn's Disease. In: Evidence-based Implant Dentistry and Systemic Conditions. New Jersey: John Wiley and Sons Ltd, USA; 2019. 13-7 p.

12. Chuang SK, Wei LJ, Douglass CW, et al. Risk factors for dental implant failure: A strategy for the analysis of clustered failure-time observations. J Dent Res. 2002;81(8):572-7.

13. Sharma A, Rahul GR, Poduval ST, et al. Assessment of various factors for feasibility of fixed cantilever bridge: A review study. ISRN Dent. 2012;2012:259891.

14. Ihde S, Ihde AA. Cookbook Mastication. Munich: Intl Implant Foundation Publishing, Germany; 2015.

15. Abraham CM. A Brief Historical Perspective on Dental Implants, Their Surface Coatings and Treatments. Open Dent J. 2014;8:50-5. 
www.yumedtext.com | October-2019

16. Smeets R, Stadlinger B, Schwarz F, et al. Impact of Dental Implant Surface Modifications on Osseo-integration. BioMed Res Int. 2016;2016:6285620.

17. Zitzmann NU, Berglundh T. Definition and prevalence of peri-implant diseases. J Clin Periodontol. 2008;35(8):28691.

18. Koldsland OC, Scheie AA, Aass AM. Prevalence of peri-implantitis related to severity of the disease with different degrees of bone loss. J Periodontol. 2010;81(2):231-8.

19. Renvert S, Polyzois I, Claffey N. How do implant surface characteristics influence peri-implant disease? J Clin Periodontol. 2011;38:214-22.

20. Ferreira SD, Martins CC, Amaral SA, et al. Periodontitis as a risk factor for peri-implantitis: Systematic review and meta-analysis of observational studies. J Dent. 2018;79:1-10.

21. Papathanasiou E, Finkelman M, Hanley J, et al. Peri-Implant Mucositis and Peri-Implantitis: A Survey of Periodontists in the United States. J Periodontol. 2016;87(5):493-501.

22. Baron M, Haas R, Dörtbudak O, et al. Experimentally induced peri-implantitis: A review of different treatment methods described in the literature. Int J Oral Maxillofac Implants. 2000;15(4):533-44.

23. Esposito M, Coulthard $\mathrm{P}$, Thomsen $\mathrm{P}$, et al. The role of implant surface modifications, shape and material on the success of osseo-integrated dental implants: A Cochrane systematic review. Eur J Prosthodont Restor Dent. 2005;13(1):15-31.

24. Lee DW. Periodontitis and dental implant loss. Evid Based Dent. 2014;15(2):59-60.

25. Wadhwa B, Jain V, Bhutia O, et al. Flapless versus open flap techniques of implant placement: A 15-month followup study. Indian J Dent Res. 2015;26(4):372-7.

26. Lazarov A. Immediate Functional Loading: Results for the Concept of the Strategic Implant®. Ann Maxillofac Surg. 2019;9(1):78-88.

27. Ihde S, Ihde AA. Diagnostics and treatment plan for the work with the strategic implant ${ }$. Munich: Intl Implant Foundation Publishing, 2017. 